\title{
LA LEGITIMIDAD DE PROXIMIDAD: UNA APROXIMACIÓN EMPÍRICA, EN LA CIUDAD DE BARRANQUILLA*
}

LEGITIMACY OF PROXIMITY: AN EMPIRICAL APPROACH

IN BARRANQUILLA CITY

JOSÉ ENRIQUE TERÁN SERNA*

\section{R E S U M E N}

A la luz de los procesos de reconfiguración del sistema capitalista en los 90's (globalización) y de las transiciones democráticas que se derivan de ello, este análisis pretende develar, que principios constitutivos de la legitimidad de proximidad caracterizan los periodos de gobernanza 2008-2011 y 2016-2019 de la administración del alcalde Alejandro Char y en la misma línea, determinar si esta nueva forma de gobernanza ha creado o consolidado algún tipo de dispositivo participativo, para la toma de decisión en los asuntos públicos de la ciudad. Al mismo tiempo se determinará, si la figura participativa de cabildos abiertos implementada en el año 2016 por el alcalde en mención, se configura como un dispositivo participativo provisto de deliberación, autonomía y cogestión entre ciudadanía-Estado, o si se reduce a una mera figura consultiva mediante la cual, la administración pública se abastece de insumos para legitimar su actuación política en la esfera pública.

PALABRAS CLAVE: Legitimidad por proximidad, cabildos abiertos, dispositivos participativos, innovación democrática.
A B S T R A T T

In the light of the processes of reconfiguration of the capitalist system in the 1990s (globalization) and the democratic transitions that derive from it, this analysis aims to reveal which constitutive principles of proximity legitimacy characterize the periods of governance 20082011 and 2016-2019 of the administration of Mayor Alejandro Char and in the same line, determine if this new form of governance has created or consolidated some type of participatory device, for decision-making in public affairs of the city. At the same time, it will be determined if the participatory figure of open councils implemented in 2016 by the mayor in question, is configured as a participatory device provided with deliberation, autonomy and co-management between citizenshipState, or if it is reduced to a mere figure consultative by means of which, the public administration is supplied with inputs to legitimize its political performance in the public sphere.

KEYWORDS: Legitimacy by proximity, open chapters, participatory devices, democratic innovation.

\footnotetext{
*Este artículo es resultado de un trabajo de investigación presentado para el curso "Sociedad Civil y Participación" en el marco de la maestría de investigación en Estudios Urbanos de la Facultad Latinoamericana de Ciencias Sociales Flacso-Ecuador

** Maestro de investigación en Estudios Urbanos. Facultad Latinoamericana de Ciencias Sociales Sede Ecuador, Quito - Ecuador. Avenida Diego de Almagro. Código Postal: 170201, Quito - Ecuador. Correo eletrónico: Jteran_10@hotmail.com
} 


\section{INTRODUCCIÓN}

El elitismo democrático se erigió después de la segunda guerra mundial, como el modelo político "ideal", sobre el cual, las naciones del mundo debían guiar sus gobiernos para garantizar el bien común de la sociedad. Este nuevo modelo político promulgaba que, solo mediante las decisiones "racionales" de las elites y la representación política, la sociedad podía llegar a un consenso común que garantizará una voluntad general, que estuviera por encima de los intereses particulares de algunos grupos de la sociedad civil (Avritzer, 2002, p.12). Según la teoría del elitismo democrático inevitablemente esto debía ser así, porque se consideraba a la masa popular como un grupo "irracional" y "emocional" incapaz de anteponer sus intereses particulares, y poder consolidar una voluntad general que beneficie a todos por igual. Solo mediante el voto popular y la elección de las elites políticas como representantes "racionales" de la sociedad civil, este objetivo esencial se cumpliría a cabalidad (Avritzer, 2002).

Sin embargo, la teoría del elitismo democrático hacia los años 70`s, evidenció una vez más, que los postulados teóricos siempre son insuficientes para aprehender una realidad dada. La supuesta "racionalidad" de las elites en América Latina, muchas veces coadyuvó a consolidar regímenes autoritarios (dictaduras) y solo fue mediante los movimientos de resistencias invocados por la sociedad civil -la masa irracional- que se logró en el continente, restablecer las reglas esenciales del orden democrático y con ello, la competencia de la élites en el sistema democrático (Lechner, 1996). A partir de los anteriores acontecimientos, nuevos grupos sociales se integrarán y transformarán la antigua acotada y reducida relación Estado-sociedad civil, por una capaz de ampliar el espectro participativo de la democracia.

Dos nociones de sociedad civil surgen en el seno de este escenario: una relacionada directamente con los principios liberalistas de la teoría neoliberal -libertad individual, liberalización del mercado, el mercado como ente regulador de la sociedad a través de la oferta y la demanda- y otra de corte comunitarista, que reivindica la acción colectiva como instrumento esencial, para contrarrestar las lógicas del sistema capitalista (Lechner, 1996). Con la puesta en escena de estas dos nociones de sociedad civil en América Latina, y la reconfiguración 
del sistema capitalista en 1990, se difumina en el continente, la creación de distintos dispositivos participativos a escala local (Nardacchione, Annunziata, Carmona 2011) que resignificaron la democracia misma, gracias a nuevos proyectos democráticos que procuraron: la ampliación y generalización de derechos, el reconocimiento, la voz y voto, así, como la construcción de espacios de deliberación y decisión, a ese segmento mayoritario de la población que históricamente, había estado excluido e invisibilizado en la toma de decisiones (Dagnino, 2006).

Según Danino (2006), estos nuevos proyectos también construyen nuevos discursos políticos que reivindican a la sociedad civil, como un eje esencial y central de la democracia en el continente. Algunos discursos buscan incorporar una participación ampliada de la sociedad civil en la toma de decisiones y otros solamente lo incorporan en el lenguaje político, la idea de la participación como un método que les garantice su permanencia en el poder, y legitimen sus actuaciones políticas.

No obstante, con la entrada en escena de nuevos actores en los años 90's al sistema democrático en América Latina, se cuestionó en primera instancia, las capacidades reales que tenía el Estado -gestión y gobierno- para garantizar la participación de los mismos en la esfera pública. Este cuestionamiento se concentró en reformar la estructura institucional y el aparato del Estado, para (re)construir tanto las prácticas y las formas existentes de procesar las demandas sociales. Estas reformas modernizaron el aparato estatal y crearon la "ilusión” de una nueva era, donde la sociedad civil, se despojaba de su histórico rol pasivo en la toma de decisiones y adquiría uno más activo, que deliberaba sobre todos los asuntos de lo público (Nardacchione, Annunziata, Carmona, 2011).

Este nuevo escenario, conllevó a que tanto la esfera local como la participación en la política local, tomaran un rol predominante en la construcción y deliberación de lo público. La nueva gestión pública indistintamente de su orientación política e ideológica, permitió una mayor participación -ampliada o reducida - de la ciudadanía y nueva formas de deliberación -informativos, consultas, cogestión, (co)gobernabilidad- en escenarios institucionales donde se debatía la construcción de lo público (Nardacchione, Annunziata, Carmona, 2011). La concreción de esta nueva gestión pública se traduce en procesos de innovación política -presupuesto participativo, proyectos de descentralización en la toma de decisiones públicas, consejos comunales, etc- o en su defecto, nuevas 
formas de gobernanza -legitimidad de proximidad- que muchas veces, busca hacer más eficiente y legitima la acción político-administrativa del Estado, sin que exista una exclusión explicita entre ambas.

En este contexto de innovación democrática, Dagnino (2006) y más específicamente en relación a las nuevas formas de gobernanza, este análisis se propone develar que principios constitutivos de la teoría de la legitimidad de proximidad -profundización de la cercanía (care) y la proximidad presentada como política de lo "eficiente" (eficientismo)-(Rosanvallone, 2009; Nardacchione, Annunziata, Carmona, 2011), se ponen de manifiesto durante los periodos de gobernanza 20082011 y 2016-2019 del alcalde Alejandro Char. Así mismo, este análisis identificará, si la legitimidad de proximidad implementada como modelo de gobernanza por el alcalde Alejandro Char en los periodos a analizar, ha detentado en la cristalización de un dispositivo participativo, teniendo en cuenta que desde la teoría se establece que los dispositivos participativos se constituyen como la forma institucional más desarrollada de la legitimidad de proximidad (Annunziata, 2009).

Metodológicamente, este artículo se plantea una revisión de fuentes de la literatura especializada en torno al tema de la legitimidad de proximidad
(Rosanvallone, 2009; Nardacchione, Annunziata, Carmona, 2009,2011) y la revisión de fuentes secundarias: notas de prensa, informes de entidades institucionales y tesis para abordar el caso de estudio. A partir de lo anterior este análisis se propone dar respuesta a dos preguntas centrales: ¿Que principios constitutivos de la legitimidad de proximidad caracterizan los periodos de gobernanza 2008- 2011 y 20162019 del alcalde Alejandro Char? y establecer si, ¿Los cabildos abiertos implementados en el 2016 por el alcalde Alejandro Char, se constituyen como un dispositivo de tipo participativo?

En aras de dar respuesta a lo planteado, este artículo se estructura en tres momentos de análisis: primero, un acercamiento a las teorías de la legitimidad de proximidad y la innovación democrática, a partir de la revisión de la bibliografía especializada. Segundo, un análisis sobre cuáles de los principios constitutivos de la legitimidad de proximidad se manifiestan en los periodos de gobernanza analizados (2008-2011: 2016-2019). Y tercero, las conclusiones del caso en las cuales se devela, que si bien existen ciertos principios verificables de legitimidad de proximidad en los periodos de gobernanza analizados, estos no detentan en la cristalización de un verdadero dispositivo participativo. 


\section{ACERCAMIENTO TEÓRICO: INNOVACIÓN DEMOCRÁTICA Y LEGITIMIDAD POR PROXIMIDAD}

A partir de la década de los 80's, en América Latina han proliferado procesos de profundización e innovaciones democráticas, que han permitido una consolidación y resignificación de la democracia misma, gracias a nuevos proyectos democráticos que han consolidado la ampliación y generalización de derechos, reconocimiento y, voz y voto, así como también, espacios de deliberación y decisión a un segmento mayoritario de la población que históricamente estaba invisibilizado (Dagnino, 2006). No obstante, estos proyectos neoliberal y democrático-participativo que han posibilitado y dado un nuevo impulso a la democracia como tal, desde sus distintas ideologías, intereses y lógicas, están utilizando un lenguaje común que les garantice, tanto el sostenimiento de las relaciones de poder como la permanencia en la esfera del poder Estatal.

Esta disputa entre dos proyectos políticos vislumbra en una pugna aséptica de estas corrientes, sin embargo esta pugna se caracteriza por una confluencia perversa, que reviste en primera instancia el encuentro de los modelos: el democrático-participativo que surge como resistencia a los regímenes autoritarios de América Latina y el neoliberal que surge a finales de los años 80 como una apuesta política-social y económica del capitalismo. En segunda instancia, ambos modelos hacen uso de las nociones de ciudadanía, participación y sociedad civil, para consolidar su ideario teleológico (Dagnino, 2006).

Los dos proyectos desde su discurso, teoría y conceptualización, presentan diferencias en cuanto a las nociones de ciudadanía, participación y sociedad civil. En el caso del modelo democrático-participativo, su objetivo principal es ampliar los procesos democráticos superando los límites de la democracia representativa liberal (Dagnino, 2006). La noción de sociedad civil es asumida como un factor clave que posibilita una mejor toma de decisiones en aras de mantener un equilibrio en el principio de igualdad, ya que la participación activa de la sociedad, concentra objetivamente la solución a un problema social (Dagnino, 2006).

Al mismo tiempo, la participación se consolida como una herramienta capaz de (re)asignar y (re)cuperar el rol protagónico del Estado, como un ente autónomo, capaz de velar por lo público. En síntesis, el Estado vuelca su 
accionar y recupera su rol, garantizando con ello, un Estado mucho más preocupado por los asuntos de lo público, sin mayor intermediación del sector privado. Esto ineludiblemente, activa procesos de rendición de cuentas que buscan asegurar el mantenimiento de derechos y control social, así como la implementación de una co-gestión entre ciudadanía y Estado. La noción de sociedad civil, se circunscribe en la organización que puede emerger de la sociedad y que además presenta un alto grado de heterogeneidad ampliada e inclusiva (Dagnino, 2006).

En el modelo neoliberal, su principal objetivo se centra en la reconfiguración del sistema capitalista y en el derribamiento de las barreras que permiten la expansión del gran capital a nivel internacional (Dagnino, 2006, p.60). Esta reconfiguración del sistema capitalista, busca el traslado de las competencias estatales al sector privado $\mathrm{y}$ con ello una reducción notable de su accionar en los asuntos de lo público. Las lógicas del mercado imperan en las dinámicas estatales, provocando con ello, que la noción de ciudadanía quede reducida a un mero asunto de "cliente" o en su defecto "usuario", anulando la concepción de derechos ciudadanos, reconocimiento y poder de decisión. La sociedad civil al mismo tiempo, es vista como una fuente de información de demandas sociales y como un mecanismo que orienta la acción del Estado hacia las mismas. Esto recrea un panorama selectivo y excluyente de la población que integra la sociedad civil (Dagnino, 2006) y que se limita a ciertas organizaciones (ONG), capaces de generar cierta influencia en el Estado. Este panorama, reduce la participación de la ciudadanía en la toma de decisiones, ya que estas organizaciones junto con el Estado, crean y producen las soluciones para las problemáticas sociales.

A tenor de lo anterior, la participación de la sociedad civil queda reducida a una "participación solidaria" carente de lo público, provocando, la anulación de derechos sociales conquistados por grupos históricamente excluidos y la despolitización de las demandas sociales (Dagnino, 2006, pp.62-64).

A pesar que estos dos modelos aparezcan como excluyentes entre sí mismo, Dagnino resalta, que puede existir una (co)existencia y una confluencia perversa entre ambos modelos. El caso del presupuesto participativo en Porto Alegre refleja dicha realidad. El presupuesto participativo se concibe, como una nueva forma de participación de la democracia deliberativa, en donde las decisiones concernientes a la planificación, la ejecución, la distribución y la estructuración del gasto público de la ciudad, son tomadas en 
mutuo acuerdo y en "igualdad de condiciones" por las partes involucradas Estado y sociedad civil en los asuntos de lo "publico" (Baiocchi, 2003; De Sousa, 2004; Sintomer, 2002). No obstante, el dispositivo de innovación democrática presenta una serie de limitaciones, que permean la orientación hacia un modelo democráticoparticipativo; de hecho, es posible evidenciar la coexistencia de ambos modelos en el dicho dispositivo.

Debido a la escasez presupuestaria de Brasil, existen inconvenientes para que la sociedad civil procure mediante la deliberación, una igualdad equilibrada de las demandas sociales. Esto se encuentra estrechamente relacionado con cierto desnivel que se presenta en cuanto a la experiencia, la capacidad organizativa y capacitación que tienen ciertos grupos de la sociedad civil (Dagnino, 2006, p. 67). Esta tendencia, centra la atención Estatal a aquellas organizaciones que tiene mayor experiencia y capacidad de agencia, vinculando esta realidad a una dinámica más cercana al modelo neoliberal en donde la sociedad civil es vista como una fuente de información sobre las demandas sociales y al mismo tiempo, las que se encuentren mejor organizadas, serán capaces de consolidar la cogestión y en consecuencia solucionar ciertas demandas sociales. La prelación estatal, sobre la sociedad civil que está mejor organizada produce una serie de domesticación y reducción de la conflictividad, capaz de deslegitimar aquella sociedad civil mucho más espontánea y no organizada (Dagnino, 2006, p. 68). A pesar de que el dispositivo de PP de Porto Alegre amplié la participación de un segmento de la población civil históricamente excluida, la lógica de la configuración estructural del dispositivo, guarda cierta lógica con el modelo neoliberal, ya que produce una especie de selección y exclusión que habilita, la participación de una sociedad civil organizada, en detrimento de la sociedad civil que no lo está.

En el marco de la confluencia perversa entre el modelo neoliberal y los nuevos procesos de innovación democrática, surge también un nuevo tipo de gobernanza en la administración de lo público denominado, legitimidad de proximidad. La legitimidad de proximidad se ha erigido en América Latina, como una nueva forma de gobernanza capaz de superar, la insatisfacción creciente dejada por la democracia representativa, ya que su modelo estructural mostro una alta ineficiencia a la hora de canalizar y solucionar de manera óptima las demandas sociales surgidas en el seno de los nuevos grupos sociales de la región (Maxwell et al, 2012; Rosanvallone, 2009). 
A grosso modo, la legitimidad de proximidad es definida como esa capacidad que tiene el gobernante o el poder local de estar cerca de sus electores, mediante una relación estrecha entre "iguales", donde la empatía y la compresión del contexto inmediato permite conocer de primera mano al elegido, las necesidades sociales que aquejan a los ciudadanos (Rosanvallone, 2009, p. 248). Esta relación de cercanía con los electores supone que, las decisiones que tome el gobernante serán mucho más legitimas en la medida que no solo ha escuchado las demandas sociales sino que también ha permitido a la población, participar de manera activa en la soluciones de sus problemas cotidianos. Esta nueva forma de gobernanza también se caracteriza porque los espacios en donde se presentan estas deliberaciones, son escenarios propios -el barrio- de la realidad inmediata de los electores (Nardacchione, Annunziata, Carmona, 2011, p. 305).

Algunos principios axiológicos que definen a la legitimidad de proximidad son, las nociones de "participacionismo" y "localismo". En ellas se detenta un reconocimiento implícito por parte del gobernante hacia sus electores, en donde la experiencia particular y el conocimiento del contexto cotidiano se erigen, como un saber popular altamente capacitado para develar y proveer de información vital al gobernante, a la hora de plantear las soluciones a una problemática determinada: "El conocimiento de la cotidianidad es valorado, sirve para identificar la demanda, la necesidad. Los vecinos realizan un gran aporte a la gestión desde este punto de vista” (Annunziata, 2011, p. 68).

La literatura académica también señala, otras características que acompañan a la legitimidad de proximidad: 1) la legitimidad de proximidad no solo se remite a la construcción de políticas públicas, también es una forma de gobierno que acompaña a una administración pública o local, 2) ha sido utilizada por representantes políticos de distintas escalas espaciales (local, regional, nacional, etc.) como una estrategia política, y 3) no es implementada por una ideología o partido político especifico. Todas las ideologías y partidos políticos han utilizado indistintamente la legitimidad de proximidad, como una herramienta para la legitimar sus gobiernos (Annunziata, 2011).

No obstante, algunos autores resaltan algunas críticas que presenta la legitimidad de proximidad. La primera de ellas se centra en que lo que esencialmente busca la legitimidad de proximidad a través de la implementación del dispositivo participativo, es solamente escuchar a la gente, más que 
construir una deliberación racional de las decisiones y en segundo lugar, que estos dispositivos participativos a pesar de ser espacios abiertos a la deliberación pública, siempre son regulados por la lógica del poder estatal (Annaunziata, Carmona y Nardacchione, 2009), por lo tanto, no es extraño encontrar una lógica top down en la toma de decisiones. Al mismo tiempo, estos dispositivos no nacen como exigencias de movilizaciones sociales, sino que son implementados por la lógica estatal, quedando entre dicho, la posible "autonomía" que tendría la sociedad civil al interior del dispositivo (Annunziata, 2011).

Otras miradas más positivas surgen desde la academia cuando se analiza empíricamente y a profundidad, las dinámicas que subyacen al interior de estos dispositivos participativos. A pesar de que exista cierta regulación estatal en estos dispositivos, las demandas y deliberaciones que surgen en el seno de la sociedad civil pueden más o menos influir, en las decisiones que tome la administración pública e incuso muchas veces, disputar en igualdad de condiciones ciertas decisiones concernientes a lo público (Ramírez, 2008, p.64) so pena, de ser castigados electoralmente en la siguiente contienda electoral si hacen caso omiso a los intereses surgidos en el seno del dispositivo participativo. También este tipo de implementación de dispositivos participativos, incrementa la posibilidad de un mayor control político y social por parte de la ciudadanía hacia sus gobernantes (Avritzer, 2002).

\section{CARACTERIZACIÓN DE LOS PERIODOS DE GOBERNANZA DE ALEJANDRO CHAR EN EL MARCO DE LA TEORÍA DE LA LEGITIMI- DAD POR PROXIMIDAD.}

\subsection{Primer periodo de Gobernanza del Alcalde Alejandro Char 2008-2011.}

Previo a la elección de Alejandro Char 2008-2011 como alcalde la ciudad, Barranquilla presentaba un escenario bastante desalentador en muchos aspectos de la vida social, económica y política. El proceso de desindustrialización en la ciudad se presenta alrededor de los años 80`s, transformando radicalmente su modelo económico hacia uno -tercerización- mucho más acorde con las lógicas de la globalización. Esto trajo consigo un crecimiento exponencial de desempleo e informalidad laboral en la ciudad (Roa, 2013). Al mismo tiempo, la administración pública de la ciudad se encontraba en manos de las elites políticas, acusadas de ser ineficientes y 
corruptas y donde los bienes del Estado, eran negociados para sus propios intereses (Salcedo, 2000). A tenor de este escenario, la proliferación de barrios subnormales (Roa, 2013) y la mercantilización de los servicios públicos domiciliarios de la ciudad, agravaron aún más el futuro de la ciudad (Varela, 2007:2012).

La elección del Alcalde Alejandro Char "supuso" para la ciudad una renovación político-administrativa de cómo se gestionaban los asuntos públicos de la ciudad. Su plan de desarrollo denominado: "Barranquilla ciudad de oportunidades" permitió en primera instancia, ordenar las finanzas públicas de la ciudad e iniciar un ambicioso proyecto de planificación urbana que buscaba mejorar el atraso real que presentaba la ciudad en materia de equipamiento urbano. Este ambicioso plan de planificación urbana abarco sectores vitales de la ciudad: como la salud, la educación, e infraestructura vial en barrios subnormales, con el único propósito de cerrar la brecha social existente en la ciudad (Alcaldía de Barranquilla 2010), con ocasión, a los malos manejos administrativos de las elites políticas locales.
A través de su plan de desarrollo, mejoró ostensiblemente el sistema de salud de la ciudad. En el planteó un nuevo modelo orientado a la construcción de P.A.S.O.S (Punto de Atención en Salud Oportunos) y C.A.M.I.N.O.S ${ }^{1}$ (Centros de Atención Medica e Integral), para mejorar la calidad de salud de los sectores más desfavorecidos y vulnerables de la ciudad. Otra estrategia que implementó dicho modelo, fue caminantes de la salud; que buscaba mediante visitas casa a casa, analizar problemas potenciales de salud pública y desarrollar programas preventivos para la población de escasos recursos. La mayoría de estos nuevos centros de salud se concentraron en las localidades -Metropolitana, Riomar, Suroccidente, Suroriente con mayor presencia en los sectores pobres, vulnerable y de escasos recursos (Pérez, 2013).

En el campo de la educación su plan de desarrollo proyectó construir 10 nuevas instituciones educativas: 7 megacolegios y 3 tres reconstrucciones en colegios existentes, con un valor aproximado de 25.774.971 mil millones de pesos (Informe de Gestión 20082011).

\footnotetext{
${ }^{1}$ En su administración se construyeron alrededor de 20 nuevas infraestructura hospitalaria entre P.A.S.O.S y C.A.M.I.N.O.S. El valor promedio de los pasos fue de 1.500 millones, mientras el de los caminos fue alrededor de los 6.500 millones de pesos.
} 
No obstante, un programa insignia que le ha generado una mayor aprobación en su imagen, es el programa "Barrios a la obra", que busca construir tramos viales en alrededor de 65 barrios vulnerables $^{2}$ de la ciudad. Su principal objetivo es mediante la construcción de vías, dignificar la condición humana para proveer mejoras en los servicios públicos y ampliar la accesibilidad la cobertura en salud y educación. Este programa además, se inscribe en la modalidad de OPC (Obras con Participación Ciudadana) donde el Estado, financia el 96\% de la obra y la comunidad provee el $4 \%$ restante (Barón, 2010). Tanto la concepción como el diseño del programa, buscar generar además una función social dirigida a la comunidad. A través de la organización barrial, la comunidad del sector realiza actividades comunitarias (bazares, rifas, venta de comida, etc.) que permiten, no solo obtener los recursos para la realización de la vía, sino también promover la cultura del ahorro, fomentar la integración comunitaria y afianzar el sentido de pertenencia al lugar (Barón, 2010).
Esto, sumado a la forma de gobernar abierta, sencilla, descomplicada e informal del Alcalde, ha permitido consolidar un lazo efectivo y afectivo entre representante y representado, donde su legitimidad, se afianza como una política de presencia y del presente (Nardacchione, Annunziata, Carmona, 2011). Su presencia radica, en la capacidad que tiene el programa no solo para convidar a la participación barrial de los habitantes del sector mediante la auto-organización de actividades comunitarias que les permita obtener los recursos para pavimentar su vía, sino también, la presencia activa y permanente del Alcalde antes, durante, y en la inauguración de todas las vías de los barrios (Alcaldía de Barranquilla, 2010). La noción del presente se evidencia, en la solución que provee el Alcalde a la población con la solución de un problema esencial como las vías, en un término no muy amplio en el tiempo. Varios son los elementos que identifican la gobernanza del Alcalde Alejandro Char como un gobierno que legitima su acción política y pública, mediante

\footnotetext{
${ }^{2}$ Las Américas, San Luis, Santa María, Santo Domingo De Guzmán, Siete De Abril, Unión, Bella Arena, Carrizal, Ciudadela 20 De Julio, José Antonio Galán, Las Flores, Las Granjas, Me Quejo, Villa San Carlos, Villas De San Pablo En El Corregimiento De Juan Mina, 7 De Agosto, Alex Char, California, Carlos Meisel, Ciudad Modesto, El Bosque, El Carmen, El Limón, El Rubí, El Valle, Evaristo Surdís, Girasoles, Kalamari, Kennedy, La Chinita, La Esmeralda, La Luz, La Manga, La Paz, La
}

Pradera, Las Estrellas, Los Olivos, Nueva Colombia, Por Fin, Villa San Pedro, Villa Del Rosario, Los Rosales, Cuchilla De Villate, El Bosque, La Chinita, La Playa, La Sierra, La Sierrita, La Victoria, Las Malvinas, Las Nieves, Las Palmas, Lipaya, Rebolo, San Felipe, San Isidro, San Nicolás, San Salvador, Santuario, Simón Bolívar, Villa Blanca, Villa Sevilla, Villa Flor, Villate, Colinas Campestres. 
la legitimidad de proximidad. La primera de ellas, es la concerniente a la noción de Care. Con su presencia en los barrios donde se inauguran vías, manda un mensaje claro de que él, se está "ocupando de sus asuntos y se preocupa por ellos” (Rosanvallone, 2009, p. 258) tal y como lo expresan algunos habitantes: "por fin un alcalde mira nuestras comunidades", "Este gobierno está mirando para Rebolo y sabemos que va a seguir mirando por las comunidades necesitadas de toda Barranquilla" (Revista institucional 2009, pp. 11-12). Esta noción de Care, también permite un acercamiento simbólico entre representante y representado enmarcado en un "localismo" (Nardacchione, Annunziata, Carmona, 2011, p. 306) real y fáctico, como lo es la visita del alcalde durante el proceso de construcción e inauguración de la vías.

Un segundo elemento que se identifica como característica de la legitimidad de proximidad en el Alcalde Alejandro Char, es el del "participacionismo". Con su visita permanente a los barrios, el alcalde escucha al ciudadano de a pie y toma en cuenta su experiencia y vivencia personal. Además con el diseño del programa "Barrios a la obra" invita al ciudadano de a pie no solo a "opinar, discutir y participar" (Nardacchione, Annunziata, Carmona, 2011) en las decisiones que se tomen para mejorar la calidad del barrio, sino también, coadyuvar mediante la auto-organización de eventos comunales, los recursos que ayuden a construir una obra tan esencial para un barrio como son las vías.

Sin embargo, la forma de gobernanza de legitimidad de proximidad del alcalde Alejandro Char, no detenta claramente en un dispositivo participativo. El programa "Barrios a la obra" no garantiza una participación efectiva de deliberación. En el solo se observa una participación incipiente, ya que las decisiones pasan por el ejecutivo y la población solo "participa" proveyendo, un $4 \%$ del valor total de la obra sin llegar a decidir y ejecutar dicho gasto. Básicamente, este programa está orientado para constituirse como un instrumento de insumo y consulta que mejoré, la eficacia de la política (Isunza, 2006: Nardacchione, Annunziata, Carmona, 2011) de pavimentación de vías, de la administración pública en barrios periféricos y vulnerables de la ciudad. Esta poca participación ciudadana por parte de la sociedad civil en la aprobación, planificación y ejecución del gasto público, imposibilita una verdadero proceso de participación y deliberación en los asuntos de lo público, máxime; si a esto se suma, que la contratación pública de la ciudad en la primera y se- 
gunda administración del Alcalde Alejandro Char, el $80 \%$ del presupuesto público se encuentra concentrado en tres empresas -Valorcom, A construir, Donado Arce \& compañía- que representan, los intereses políticos y económicos de las elites de la ciudad (Velásquez, 2016).

La inexistente (co)gobenanza entre la sociedad civil y la administración pública mediante la no implementación de un dispositivo participativo permite, que no exista una influencia o una disputa en igualdad de condiciones a la hora de definir y decidir, los asuntos públicos de la ciudad (Ramírez, 2008). Lo que realmente se evidencia, es que la redistribución del gasto público, está altamente concentrada en pocas familias que representan las elites políticas y locales de la ciudad. Esto se constituye en un gran óbice para el cometido esencial de toda administración pública, que siempre busca cerrar la brecha social de la población más vulnerable.

\section{CUADRO 1. CONTRATACIÓN PÚBLICA (2008)}

\begin{tabular}{|c|c|c|}
\hline EMPRESA & VALOR DEL CONTRATO & $\begin{array}{c}\text { CONTRATOS ADJUDI- } \\
\text { CADOS }\end{array}$ \\
\hline VALORCON & $\$ 348.257 .033 .137$ & 11 \\
\hline A CONSTRUIR SA & $\$ 269.297 .289 .494$ & 10 \\
\hline $\begin{array}{c}\text { DONADO ARCE \& COMPA- } \\
\text { ÑÍA SAS }\end{array}$ & $\$ 88.446 .669 .318$ & 10 \\
\hline $\begin{array}{c}\text { GENERACIÓN COLOMBIA } \\
\text { SA }\end{array}$ & $\$ 22.326 .873 .620$ & 10 \\
\hline $\begin{array}{c}\text { EDGARDO NAVARRO VI- } \\
\text { VES }\end{array}$ & $\$ 385.208 .710 .452$ & 9 \\
\hline $\begin{array}{c}\text { RICARDO CHAMS Y CIA } \\
\text { SIGNO } 21 \text { SAS }\end{array}$ & $\$ 13.666 .767 .797$ & 8 \\
\hline $\begin{array}{c}\text { MAYORDOMIA Y SERVI- } \\
\text { CIOS LIMITADA MAYOR- } \\
\text { DOMIA LTDA }\end{array}$ & $\$ 63.406 .863 .696$ & 6 \\
\hline $\begin{array}{l}\text { CONSTRUCCIONES E IN- } \\
\text { VERSIONES BETA LTDA }\end{array}$ & $\$ 305.215 .607 .141$ & 6 \\
\hline CARLOS VENGAL PÉREZ & $\$ 145.502 .646 .853$ & 6 \\
\hline COINSES SA & $\$ 172.102 .979 .935$ & 4 \\
\hline
\end{tabular}




\subsection{Segundo periodo de gober- nanza de Alejandro Char: los cabildos abiertos (2016).}

La legitimidad de proximidad implementada por el alcalde Alex Char en su primera administración le significo ser designado en el 2011, como el mejor Alcalde de Colombia a nivel nacional. Citado premio fue otorgado por la Organización "Colombia Líder", que se encuentra conformada por grupos de la sociedad civil y el sector privado. Esta corporación exalta la labor y los logros de los gobiernos locales en el marco de una gestión pública con excelencia (FMC 2011). No obstante, el mayor redito político alcanzado por su legitimidad de proximidad, fue dejar en manos de la administración pública, a su más ferviente y cercana colaboradora Elsa Noguera de la Espriella quien fue elegida, como alcaldesa de la ciudad en el periodo de 20122015 y que se desempeñó, como Secretaria de Hacienda en su primer mandato. Su gobernanza con ciertas especificidades y particularidades se concentró, en emular el modelo de gobernanza de la legitimidad de proximidad implementada por el anterior alcalde. Bajo este escenario favorable de continuismo de un proyecto político de la elite local, es que el exalcalde Alejandro Char decide postularse nue- vamente, como candidato a la alcaldía, siendo elegido para gobernar la ciudad durante el periodo de 20162019.

Este reconocimiento y el continuismo alcanzado por su proyecto político, evidencia otro de los principios teóricos constitutivos de la legitimidad de proximidad: "el eficientismo"; muy característico de la cercanía y la escucha directa a las necesidades de la ciudadanía, que desde la teoría se presenta como "la capacidad de identificar problemas específicos, dar soluciones específicas e involucrar a los actores directos" (Nardacchione, Annunziata, Carmona, 2011). Una muestra de esta cercanía y escucha directa a los problemas de la ciudadanía en la gestión del alcalde Alejandro Char fue la implementación de los cabildos abiertos. En sus primeros días de mandato decide junto con el Concejo Distrital de la ciudad, implementar el mecanismo de cabildos abiertos, para condensar las demandas sociales que surgen en el seno de la sociedad civil y que le permitan construir el plan de desarrollo "Barranquilla capital de vida" 20162019, mucho más acorde, a los problemáticas sociales que presenta la ciudad. Estos cabildos fueron organizados en 15 sesiones descentralizadas, en las cinco localidades que integran 
la ciudad de Barranquilla (Publimetro, 2016). Según el alcalde, este es una nueva modalidad sin precedentes de participación ciudadana, ya que estos cabildos abiertos se desarrollaron durante 42 días, con 11 grandes reuniones en las cinco localidades con público general, y 4 tertulias o foros especializados con públicos específicos (Zona Cero, 2016).

\section{FIGURA 1. DIAGRAMA DE ACTIVACIÓN DE UN CABILDO ABIERTO}
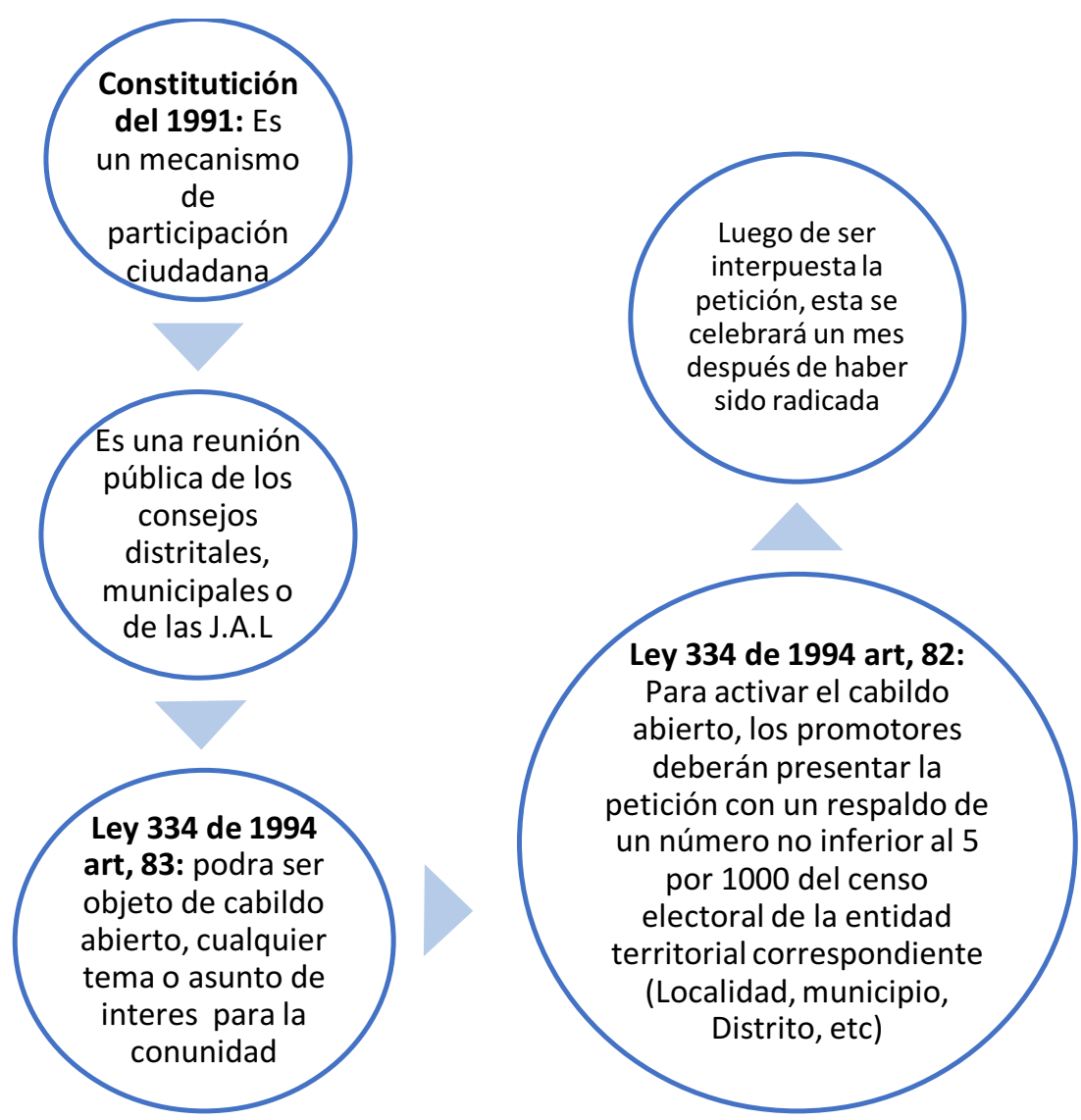

Fuente: Alvarado Beltrán, 2014; Registraduría Nacional del Estado Civil.

Sin embargo, la utilización de este mecanismo de participación ciudadana por parte del Alcalde y el Concejo Distrital de la ciudad, presenta muchas contradicciones con sus principios conceptuales y el verdadero propósito, con que fue concebida en la Constitución Política de Colombia de 1991. La primera contradicción es la que surge a 
nivel conceptual. Si bien los cabildos abiertos $^{3}$ son un mecanismo de participación ciudadana, estos hacen parte de los instrumentos con que cuenta la sociedad civil para realizar un proceso de control -accountability societal institucional- sobre la gestión y decisiones que toman las entidades estatales, sobre el bien general de una comunidad (Abal, 2009).

Una segunda contradicción que emerge con la invocación hecha por el alcalde de los cabildos abiertos en la ciudad, es la concerniente a los propósitos con que fue concebida en la Constitución. Según la Constitución Política de Colombia de 1991 en su artículo 103 se estipula que son mecanismo de participación del pueblo en ejercicio de su soberanía: el voto, el plebiscito, el referendo, la consulta popular, el $\mathrm{ca}$ bildo abierto, la iniciativa legislativa y la revocatoria de mandato. Así mismo, el cabildo abierto se concibe como un instrumento mediante el cual, la ciudadanía hace uso, para ejercer control político frente a las decisiones que tomen las instituciones estatales y los funcionarios públicos (Cogollos \& Ramírez, 2007).
Una última y tercera contradicción que emerge con respecto a la invocación hecha por el alcalde para convocar a cabildo abierto ${ }^{4} \mathrm{y}$ construir el plan de desarrollo "Barranquilla Capital de Vida”, es la concerniente al del procedimiento legal. Según la Ley Estatutaria de Participación Ciudadana 334 de 1994, en su artículo 28 establece: que para que se invoque a cabildo abierto este deberá contar con al menos el respaldo del $5 \%$, del censo electoral correspondiente a la entidad territorial. Además, el artículo 81 de la presente ley establece: que esta se debe presentar en sesiones ordinarias del concejo municipal, distrital o juntas administradoras locales. $\mathrm{Al}$ mismo tiempo, el artículo 83 de la presente ley resalta: que cualquier asunto de interés comunitario puede ser objeto invocación para la instauración del cabildo abierto.

En síntesis, la legitimidad de proximidad implementada por el alcalde Alejandro Char en el 2016 a través de los cabildos abiertos, no detento en la cristalización e institucionalización de un dispositivo participativo, capaz de deliberar y decidir los asuntos de lo pú-

\footnotetext{
${ }^{4}$ Hasta la fecha y según datos de la Registraduría Nacional del Estado Civil, la ciudad de Barranquilla nunca ha iniciado la implementación de un cabildo abierto. http://www.registraduria.gov.co/-Cabildo-abierto$\underline{\text { html }}$

\footnotetext{
${ }^{3}$ Según datos de la Registraduría Nacional del Estado Civil en Colombia, se ha realizado 89 cabildos abiertos: Santander (18), Boyacá (18), Cundinamarca (14) y Antioquia (10), Nariño (5), Tolima (5), Bolívar (5), Quindío (3), Valle (2), La Guajira (2), Valle del Cauca (1), Bogotá (1), y Cauca (1). 44 han sido aprobados y 3 no han sido aprobado, el resto está en trámite o aún no se ha consolidado su votación.
} 
blico de la ciudad por parte de la ciudadanía. Este dispositivo participativo consagrado en la constitución política de 1991 fue utilizado por parte del alcalde Alejandro Char tan solo, como una herramienta meramente consultiva, para conocer de primera mano las necesidades de la población civil. Ni su invocación, ni su puesta en marcha como mecanismo de participación ciudadana, puede considerarse, bajo el periodo de gobernanza del alcalde Alejandro Char (20162019), como la forma institucional más desarrollada de la legitimidad de proximidad (Annunziata, 2009). De hecho, la "participación" ciudadana de la sociedad civil que se dio al interior de este dispositivo participativo no se puede entender equívocamente, como que "participar" sea "decidir", y que la acción de "deliberación" se entienda; como ese acto reflexivo mediante el cual la sociedad civil, ordena, planifica y ejecuta, los asuntos de lo público en una lógica de (co)gobenanza horizontal con la institucionalidad. Para el presente caso y dado las evidencias empíricas, "deliberar" tan solo significó, "ser consultado" (Nardacchione, Annunziata, Carmona, 2011).

\section{CONCLUSIONES}

La legitimidad de proximidad puede constituirse como una herramienta esencial para orientar una actuación pública efectiva de los gobernantes, hacia su electorado. Su apertura al dialogo, la empatía, la idea de escuchar al otro, de reconocer su percepción como válida, presenta un impacto positivo tanto en la imagen de aprobación como en la gestión pública de los gobernantes. Resulta innegable cuestionar los grandes logros que alcanzó la legitimidad de proximidad en el presente caso. En el primer mandato del Alcalde Alejando Char quedó demostrado, que se condensó y articuló verdaderamente la actuación de la administración pública, hacia los intereses y las necesidades del electorado, sin embargo, esto no derivó en la consolidación de un dispositivo participativo, capaz de deliberar sobre todos los asuntos de lo público.

Se advierte, que si la legitimidad de proximidad ejercida por el alcalde Alejandro Char no se cristaliza en la constitución de un dispositivo participativo permanente, se corre el riesgo de que esta sea utilizada para legitimar únicamente la actuación pública de un gobierno, sin que esta necesariamente derive, en una democracia muchas más deliberativa, capaz de establecer una relación directa socio-estatal de cogobierno en los asuntos públicos. El estudio de caso demostró igualmente, que bajo la puesta en marcha de la le- 
gitimidad de proximidad sin la cristalización en un dispositivo participativo permanente, esta también, puede ser cooptada y puesta al servicio de los intereses de una elite local y concentrar el poder público, en manos de un único proyecto político hegemónico. $\mathrm{Al}$ mismo tiempo queda claro, que en el presente caso "participar" no significa "decidir", ni "deliberar" se tradujo en un acto de intercambio racional orientado al entendimiento (Nardacchione,Annunziata, Carmona, 2011). Lo que sí es evidente es que bajo el contexto del presente caso, la implementación de la legitimidad de proximidad está siendo utilizada más con una intensión de consulta, que como un instrumento deliberativo inherente a la esfera pública.

El presente análisis empírico también demostró, que la legitimidad de proximidad implementada por el alcalde Alejandro Char en sus dos periodos de gobernanza (2008-2011 y 2016-2019) está siendo utilizada como un mecanismo efectivo de consulta sobre las verdaderas necesidades que aquejan a la población, sin embargo, al no cristalizarse en un dispositivo participativo de deliberación y decisión, la democratización de los recursos públicos de la ciudad, están quedando única y exclusivamente en manos de las elites políticas y empresariales, sin que esto redunde verdaderamente, en una proceso de mayor equidad y justicia social que permita superar los altos niveles de pobreza que presenta la ciudad.

\section{REFERENCIAS BIBLIOGRÁFICAS}

Alvarado Beltrán, A. (2014). El cabildo Abierto en Colombia. Revista de derecho UNED, 14,79-99.

Abal Medina, J. (2009). La crisis de representación y el control sobre los representantes. En Participación y control ciudadanos: El funcionamiento de los mecanismos institucionales electorales y societales de Accountability en la Argentina (pp.17-38). Buenos Aires: Prometeo.

Alcaldía Distrital de Barranquilla. (2009). Es por ti Barranquilla. Revista Institucional, 4, 3-43.

Alcaldía Distrital de Barranquilla. (2010). El Alcalde. Recuperado de http://www.barranquilla.gov.co/acercade-la-entidad/la-alcaldesa

Alcaldía Distrital de Barranquilla. (2010). Con el Alcalde y la Reina, estreno de vías a ritmo de Carnaval. Recuperado de http://www.barranquilla.gov.co/in-

dex.php?option=com_content\&view=article\&id $=2244 \% 3 \mathrm{ACon}+\mathrm{el}+\mathrm{Al}-$ calde+y+la+Reina\%2C+estreno+de+v\%EDas+a+ritmo+de+Carnaval\&catid $=57 \% 3$ Anoticias\&ltemid=157\&lang=es

Alcaldía Distrital de Barranquilla. (2010). Informe de Gestión Completo 2008-2011. Recuperado de http://www.sedbarranquilla.gov.co/sed/documents/InformeGestion2011.pdf 
Annunziata, R, Carmona, R y Nardacchione, G. (2009). Reflexiones sobre la democracia local: políticas de apertura de la gestión, de participación ciudadana y de deliberación pública. En $1^{\circ}$ Seminario Internacional Participación ciudadana y políticas públicas: miradas desde la sociedad y desde el Estado. Buenos Aires: Instituto del Conurbano.

Annunziata, R. (2011). Proximidad", representación y participación. El Presupuesto Participativo en Argentina. Íconos: Revista de Ciencias Sociales, (40), 57-70.

Avritzer, L. (2002). Democracy and Public Sphere in Latin America, New Jersey: Princeton University Press. (Capítulol y 5), pp. 11-36 y 103-134.

Barón Orozco, F. (2010). Impacto socio jurídico del programa barrios a la obra ejecutado por el Distrito de Barranquilla durante los años 2008-2009. (Tesis de pregrado, Corporación Universitaria Rafael Núñez).

Baiocchi, G. (2003). Participación, activismo y política: el experimento de Porto Alegre. En Fung, A y Wrigth E (Ed). Democracia en Profundidad, A., The Real Utopias Project (pp. 89-138). Bogotá: Universidad Nacional de Colombia

Cogollo, S y Ramírez, J. (2007). El camino tortuoso de la participación ciudadana. Una mirada al cabildo abierto en Bogotá. Universitas Humanística, 63: 109131.

Comunicaciones Federación Colombiana de Municipios. (2011). Premio al mejor Alcalde y Gobernador de Colombia 20082011. Federación Colombiana de Municipios (FCM), 1 de Julio.

Constitución Política de Colombia. (1991).
Dagnino, E, Olvera, A y Panfichi, A. (2006). La disputa por la construcción Democrática en América Latina. México: FCE-CIESAS-UV.

De Sousa Santos, B. (2004). Presupuesto participativo en Porto Alegre: para una democracia redistributiva. En Democratizar la democracia (pp. 360-444). FCE: México.

Lechner, N. 1996. "La (problemática) invocación de la sociedad civil". Perfiles Latinoamericanos, № 6: 131-144.

Ley Estatutaria de Participación Ciudadana 334 de 1994.

Maxwell A. Cameron, Eric Hershberg y Kenneth E. Sharpe, 2012. "La VOz institucionalizada en las democracias de América Latina" (Cap. 11), en Nuevas instituciones de democracia participativa en América Latina: la voz $y$ sus consecuencias, Cameron, Hershberg y Sharpe (editores) FLACSO: México.

Nardacchione, G, Annunziata, R y Carmona, R. (2011). Democracia local: políticas de apertura de la gestión, de participación ciudadana y de deliberación pública. En Todos Juntos, Dispositivos de participación de los gobiernos locales en la Argentina reciente. (pp. 291-315). Buenos Aires: Prometeo Libros.

Pérez, G. (2013). Accesibilidad geográfica y equidad en la prestación del servicio de salud: un estudio de caso para Barranquilla. Borradores de Economía, 770, 1-31.

Registraduría Nacional del Estado Civil, (2017). Cabildos Abiertos. http://www.registraduria.gov.co/-Cabildo-abierto-.html 
Rosanvallon, P. (2009). La legitimidad democrática. Imparcialidad, reflexividad, proximidad. Buenos Aires: Manantial.

Publimetro. (2016, 14 de enero). Concejo aprueba Cabildo Abierto para los barranquilleros. Publimetro, p.1.

Ramírez Gallegos, F. (2008). ¿El espacio público como potencia. Controversias sociológicas desde la experiencia participativa de Medellín, Iconos, 32: 6173.

Roa Barraza, W. (2013). Llanos Henríquez, Efraín. Una aproximación a la geografía histórica de Barranquilla en el siglo XX. Historia Caribe, 8, (23): 297-299.

Salcedo, L. (2000). Barranquilla, sitios de libres. En Abello, A y Giaimo, S. (Ed), Poblamiento y ciudades del Caribe Colombiano (pp. 103-150). Bogotá: Observatorio del Caribe Colombiano.

Sintomer, Y. (2002). Porto Alegre. El desafío de la democracia participativa, Quito: Centro de Investigaciones CIUDAD Abya Yala.

Varela, E. (2007). Las privatizaciones en Cartagena y Barraquilla. Un paradigma mercantilista en la gestión de los servicios públicos domiciliarios en Colombia. Pensamiento \& Gestión, 23: 209-255.

Velásquez, T. (2016, 31 de enero). Los megacontratistas de la era Char en Barranquilla. La Silla Vacía. Recuperado de http://lasillavacia.com/historia/los-megacontratistas-de-barranquilla-en-la-erachar-52921

Zona Cero. (2016, 18 de enero). En cabildo abierto se definirá Plan de Desarrollo 'Barranquilla Capital de Vida. Zona Cero.
Recuperado de http://zonacero.com/?q=politica/en-cabildo-abierto-se-definira-plan-de-desarrollobarranquilla-capital-de-vida-52638

\section{PARA CITAR ESTE ARTÍCULO:}

Terán Serna, J. (2018). La legitimidad de proximidad: una aproximación empírica, en la ciudad de barranquilla. Collectivus, Revista de Ciencias Sociales, 5(2), 60-79.

DOI: http://dx.doi.org/10.15648/Coll.2.2018.5

Recibido: 22/03/2018

Aprobado: 05/05/2018 